\title{
Geschlechterspezifische Unterschiede
}

Fragestellung: Wie häufig sind depressive Symptome im Alter und welche Risikofaktoren sind damit assoziiert?

Hintergrund: Depressive Symptome sind im Alter häufig. Während bis zu 20\% der jeweils untersuchten Probanden ab 65 solche Symptome zeigten, leiden typischerweise zwischen 1-5\% unter Symptomen, die die Diagnose einer Major-Depression rechtfertigen. Depressive Symptome sind assoziiert mit weiblichem Geschlecht, geringer sozialer Unterstützung, kognitiver oder körperlicher Beeinträchtigung, somatischen Erkrankungen und vorangegangenen Episoden einer Depression. Die Rolle anderer potenzieller Risikofaktoren, wie Bildung, Alter und finanzielle Bedingungen, konnte nicht eindeutig nachgewiesen werden. Es gibt insgesamt zu wenige Erhebungen über depressive Symptome und Risikofaktoren bei älteren Menschen, obwohl diese für die Versorgung relevant sind, so dass hieran ein besonderes Interesse besteht.

Patienten und Methodik: Die Datenbasis für die Studie wurde einem Datensatz entnommen, der anlässlich einer Befragung von 5.033 repräsentativ und zufällig ausgewählten Einwohnern in Deutschland im Alter von 14 und höher anfiel. Davon waren 1.659 Patienten 60 Jahre alt und älter. Depressivität wur-

Glaesmaer H, Riedel-Heller S, Braehler $\mathrm{E}$ et al. Age- and gender-specific prevalence and risk factors for depressive symptoms in the elderly: a populationbased study. Int Psychogeriatrics 2011; 23: 1294-300 de in Form einer Selbstbeurteilung mit der deutschsprachigen Version des Depressionsmoduls des Patient Health Questionnaire (PHQ9) erhoben. Ein Score von 0-4 wurde als unauffällig angenommen, 5-9 wurde als „depressive Symptome“, 10 - 14 als „Minor-Depression“ und 15-19 als „Major-Depression“ kategorisiert. Parallel wurde die Anzahl der chronischen, somatischen Erkrankungen erhoben und die soziale Unterstützung, wie auch verschiedene soziodemografische Variablen. Depressivität in der Form des PHQ-9-Scores war die abhängige Variable in verschiedenen linearen Regressionsmodellen.

Ergebnisse: Depressive Symptome waren bei Frauen (31,3\%) nicht signifikant unterschiedlich häufiger als bei Männern $(25,6 \%)$ über 60 Jahren. Symptome einer Major-Depression waren bei Frauen (3,3\%) häufiger als bei Männern (2,2\%), allerdings war die Prävalenz hierfür fast zehnfach niedriger als die der subklinischen Depressivität. Eine Minor-Depression lag bei 4,4 \% der Männer und 3,3\% der Frauen vor. Für Männer waren höheres Alter und geringere finanzielle Mittel signifikant mit der Äußerung depressiver Symptome assoziiert, während dies nicht für Frauen galt. Bei beiden Geschlechtern war die Anzahl chronischer medizinischer Probleme sowie schlechtere soziale Unterstützung mit einer erhöhten Wahrscheinlichkeit assoziiert, depressive Symptome zu haben. Diese beiden Faktoren waren auch diejenigen mit der stärksten Faktorladung. Nicht signifikant waren Geschlecht, Bildungsgrad und ob der Proband allein lebte.

Schlussfolgerungen: Depressive Symptome zeigen sich im Alter in einem weiten Spektrum. Schlechte soziale Unterstützung und die Auswirkungen medizinischer Probleme erscheinen als Risikofaktoren, die grundsätzlich einer Veränderung zugänglich sein sollten. Behandler sollten deswegen diese Aspekte im Rahmen eines umfassenden Behandlungsplans mit berücksichtigen.

\section{- Kommentar von Dr. Dr. Felix Wedegärtner}

\section{Ältere brauchen ein umfassenderes Assessment}

Die Autoren stellen ein kategoriales Vorgehen (keine, leichte, Minor-, Major-Depression) einem dimensionalen Vorgehen gegenüber (Anzahl der depressiven Symptome). Dies hat den Vorteil, dass sowohl die Erkrankungsprävalenz erhoben werden kann, als auch die Korrelation verschiedener Risikofaktoren mit der Schwere der Depressivität. Weil bekannt ist, dass zumindest diejenigen depressiven Bilder, die Behandlung erfahren, im mittleren Alter bei Frauen häufiger als bei Männern sind, ist es eine interessante Frage, auf welche unterschiedliche Weise sich depressive Bilder bei Männern und Frauen äußern.

Ein interessantes Ergebnis der Studie ist, dass sich bei Berücksichtigung der subklinischen Depressivität die Geschlechterprädilektion praktisch aufzuheben erscheint. Dies weist auf die wichtige Rolle des Depressionserlebens für die Zuschreibung einer klinischen Kategorie hin, das offensichtlich geschlechterspezifisch unterschiedlich ist. Ein wichtiger Aspekt, der sowohl die steigende Prävalenz depressiver Erkrankungen als auch die Geschlechterunterschiede erklären könnte. Weil soziale Unterstützung und medizinische Komplikationen so starke Auswirkungen auf die Ausprägung von Depressivität haben, empfiehlt sich bei Älteren ein umfassendes Assessment, bei dem diese individuellen Aspekte mit erhoben werden.

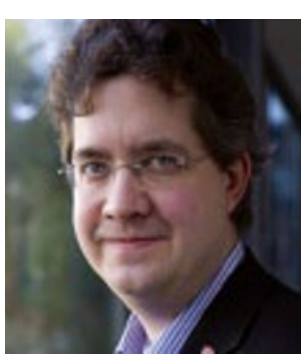

Dr. med. Dr. PH Felix Wedegärtner, Hannover

Medizinische Hochschule Hannover, Klinik für Psychiatrie, Sozialpsychiatrie und Psychotherapie, Hannover E-Mail:

wedegaertner.felix@mh-hannover.de 